\title{
Protecting-group directed stereospecific organocatalytic [3+2] cycloadditions: a facile access to chiral spirocyclic oxindoles
}

\author{
Bin Tan, ${ }^{a, b}$ Xuan Zhang, ${ }^{c}$ and Guofu Zhong*,a \\ ${ }^{a}$ College of Materials, Chemistry and Chemical Engineering, Hangzhou Normal University, \\ 16 Xuelin St., Hangzhou, ZheJiang 310 036, P. R. China \\ ${ }^{b}$ Department of Chemistry, South University of Science and Technology of China, \\ Tangchang Rd., Shenzhen, Guandong 518 055, P. R. China \\ ${ }^{c}$ Division of Chemistry and Biological Chemistry, School of Physical and Mathematical \\ Sciences, Nanyang Technological University, 21 Nanyang Link, Singapore 637 371, Singapore \\ E-mail: zgf@hznu.edu.cn
}

\section{Dedicated to Professor Pierre Vogel on the occasion of his $\mathbf{7 0}^{\text {th }}$ birthday}

DOI: http://dx.doi.org/10.3998/ark.5550190.p008.401

\begin{abstract}
An efficient organocatalytic [3+2] cycloaddition between isocyanides and methyleneindolinones, with simultaneous formation of two quaternary stereocenters, for the rapid construction of dihydrospiro[pyrrolidin-3,3'-oxindole] derivatives with high enantiopurity and structural diversity was developed. Furthermore, different protecting group on the nitrogen atom of methyleneindolenones gave rise to a different major diastereoisomer, suggesting a new avenue of great importance to medicinal chemistry and diversity-oriented synthesis.
\end{abstract}

Keywords: Asymmetric catalysis, oxindoles, spirocyclic, organocatalysis, protecting group

\section{Introduction}

The spiro[pyrrolidin-3,3'-oxindole] skeleton is commonly presented in a large number of natural products $^{1-3}$ as well as medicinally relevant compounds ${ }^{4-6}$ (Figure 1) and associated with significant biological activities. For instance, the Spirotryprostain B, isolated from the fermentation broth of Aspergillus fumigatus, has been proved to render complete inhibition of the $\mathrm{G} 2 / \mathrm{M}$ progression in mammalian tsFT210 cells. ${ }^{7}$ In light of promising bioactivities, the therapeutic potential of this attractive heterocyclic spiro motifs has led to a demand for efficient construction of spiro[pyrrolidin-3,3'-oxindole] ring system with high enantioselectivity. 
<smiles>CC[C@H]1CN2CC[C@]3(C(=O)Nc4ccccc43)C2C[C@H]1/C(=C\OC)COC</smiles>

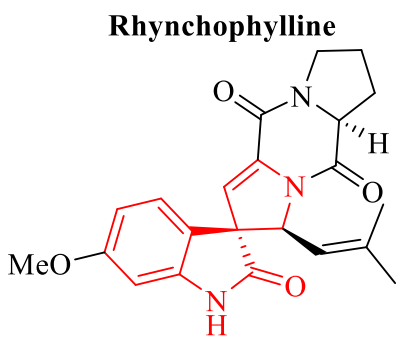

Spirotryprostain B

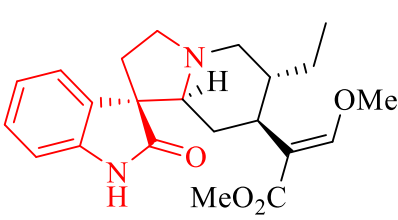

Rhynchophylline<smiles></smiles>

Spirotryprostain B

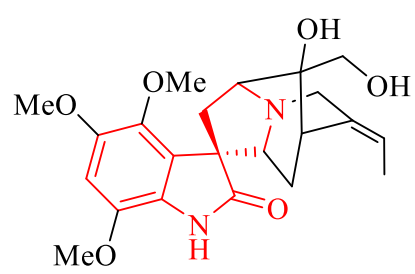

Chitosenine

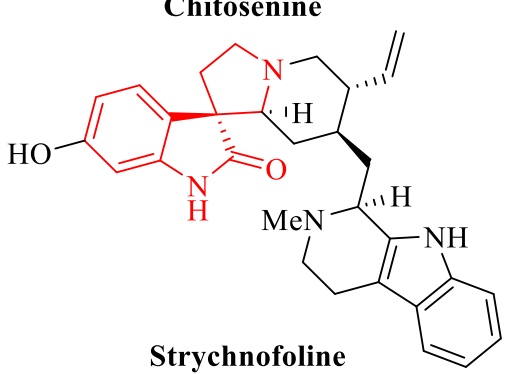

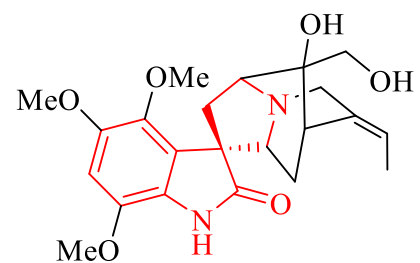

Chitosenine

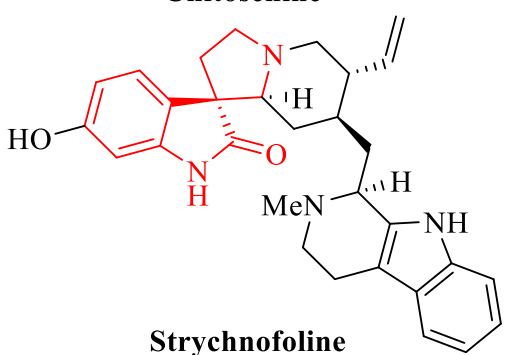<smiles>CC(C)(C)C[C@H]1N[C@H](C(=O)NC[C@H](O)CO)[C@H](c2cccc(Cl)c2)[C@@]12C(=O)Nc1cc(Cl)c(F)cc12</smiles>

Tang's Lead Compound

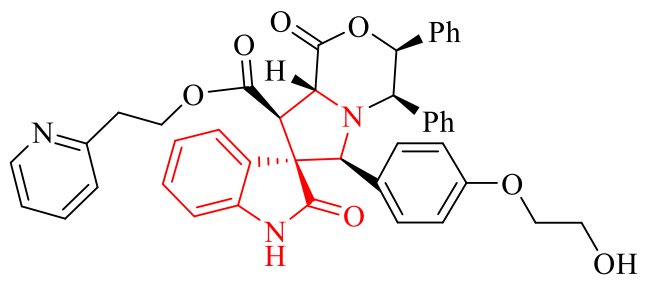

Schreiber's Lead Compound<smiles>CC(C)(C)C[C@H]1N[C@H](C(=O)NC[C@H](O)CO)[C@H](c2cccc(Cl)c2)[C@@]12C(=O)Nc1cc(Cl)c(F)cc12</smiles>

Tang's Lead Compound

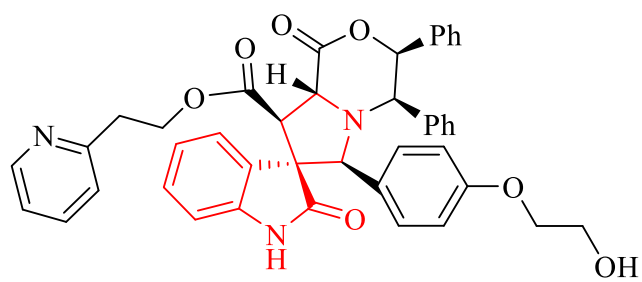

Schreiber's Lead Compound

Figure 1. Some examples of natural occurring and biologically active spirocyclic oxindoles.

Over the past several years, intensive effort has been put into the asymmetric construction of this type of spirocyclic oxindole skeletons. However, resulting from the spiro structure, only a few transformations have achieved the goal. ${ }^{8-21}$ The challenges associated with the stereocontrolled construction of spirocyclic oxindole core arise from introducing quaternary carbon stereocenter at C-3 of oxindole, ${ }^{22,23}$ which is highly sterically congested. As a result, the direct catalytic enantioselective synthesis of the spirocyclic oxindole structure with two quaternary carbon chiral centers ${ }^{24,25}$ remains a daunting challenge. 


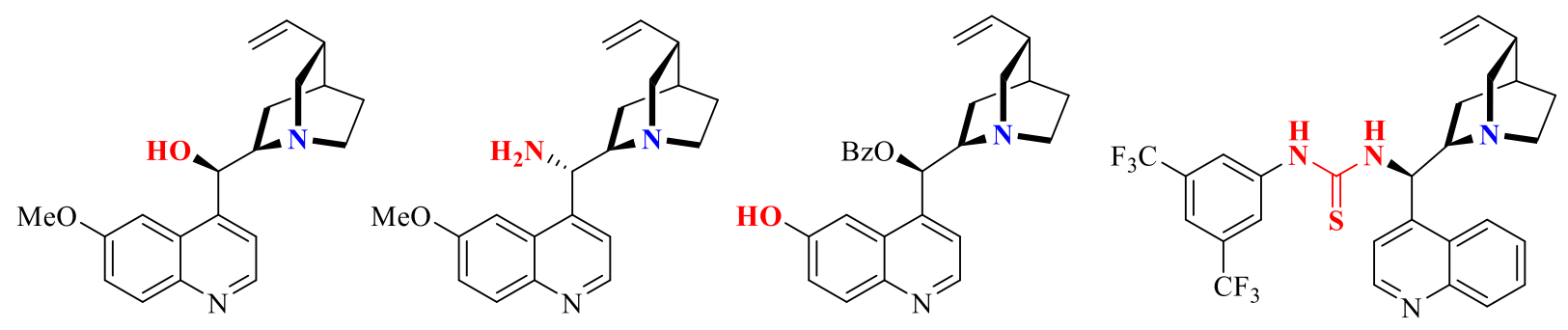

I<smiles>CCC1C[C@@H]2CCN(C1)[C@@H]2NC(=S)Nc1cc(C(F)(F)F)cc(C(F)(F)F)c1</smiles>

II

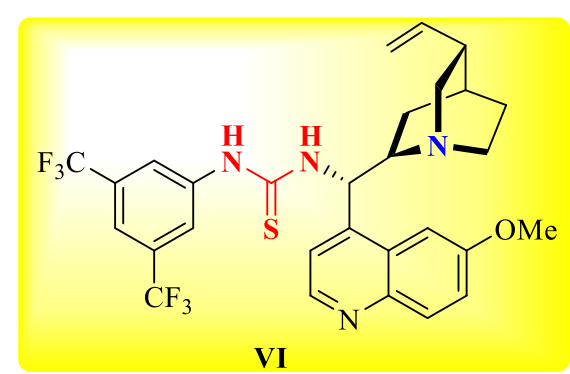

IV<smiles>C=CC1CN2CC[C@@H]1C[C@H]2[C@H](NC(=S)Nc1cc(C(F)(F)F)cc(C(F)(F)F)c1)c1ccnc2ccc(OC)cc12</smiles>

Figure 2. Structures of cinchona alkaloid derived organocatalysts.

Cinchona alkaloids ${ }^{26-37}$ and their derivatives (Figure 2) have proven to be powerful organocatalysts for various organocatalytic ${ }^{38-42}$ asymmetric C-C bond formations. Recently, isocyanide has been reported as an efficient nucleophile, ${ }^{43-46}$ as the $\alpha$-hydrogen atom is sufficiently acidic to be deprotonated by cinchona alkaloids. Inspired by this discovery, it is envisioned that the [3+2] cycloaddition of isocyanide and methyleneindolinone may be promoted by bifunctional cinchona alkaloid catalysts, leading to a direct stereoselective access to dihydrospirocyclic oxindoles, which may be easily transformed into the spiro[pyrrolidin-3,3'oxindole] derivatives (Scheme 1).

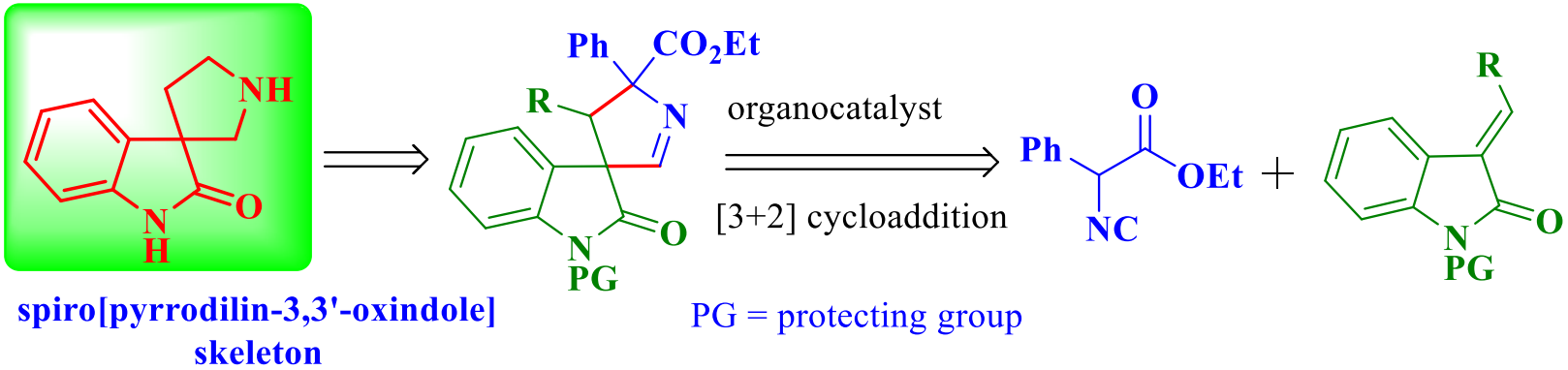

Scheme 1. Proposed strategy for construction of spiro[pyrrolidin-3,3'-oxindole] skeletons by $[3+2]$ cycloaddition between isocyanides and methyleneindolinones.

In this communication, the discovery of the first asymmetric organocatalytic [3+2] cycloaddition between isocyanide and methyleneindolinone with simultaneously formation of two quaternary and one tertiary stereocenters is reported for the rapid construction of 
dihydrospiro[pyrrolidin-3,3'-oxindole] derivatives with high enantiopurity and structural diversity. Furthermore, different protecting group gave rise to different major diastereoisomer, which suggested a new avenue of great importance to medicinal chemistry and diversity-oriented synthesis.

\section{Results and Discussion}

The initial optimization began with the addition of isocyanide 1 a (1.5 equiv) to methyleneindolinone $2 \mathbf{a}$ in $\mathrm{CH}_{2} \mathrm{Cl}_{2}$ in the presence of commercially available quinine (catalyst $\mathbf{I}$, $10 \mathrm{~mol} \%$ ) at room temperature. Although the reaction proceeded smoothly, the major product was separated from a 1.1 to 1 mixture of diastereoisomers in $43 \%$ yield with a poor enantioselectivity ( $32 \%$ ee, Table 1 , entry 1 ). Several other cinchona alkaloids were tested (catalysts II and III) under the same condition, however, the yield and selectivity were generally not good (Table 1, entries 2 and 3). Notably, a significant improvement in both diastereo- and enantioselectivity was observed when cinchona alkaloids containing thiourea scaffolds were screened (Table 1, entries 4-7). The reactions fully completed within 2 hours and the highest ee was achieved using catalyst VI, accomplished with good yield $(76 \%)$ and catalyst VII produced the opposite enantiomer with comparable ee value. A subsequent solvent screening revealed that nonpolar solvents are beneficial to this type of reaction, as slight increase in enantioselectivity and yield was observed in toluene (Table 1, entry 8). There was somewhat drop in ee when only 1.0 equiv of isocyanide 1a was used (Table 1, entry 10). Decreasing the reaction temperature prolonged the reaction time with no improvement in either enantioselectivity or yield (Table 1 , entry 11). Finally, $5 \mathrm{~mol} \%$ catalyst loading was found to be better for obtaining high yield and excellent enantioselectivity (Table 1, entries 12 and 13).

With the optimized condition in hand, the scope of Boc-protected methyleneindolinones was investigated (Table 2). The presence of both electron withdrawing group and donating group at the indolinone moiety was all tolerated to afford more than $99 \%$ ee (Table 2, entries 1-5). Methyleneindolinone derivatives bearing various substituents at the $\mathrm{C}-\mathrm{C}$ double bond also participated in the direct [3+2] cycloaddition reactions. Excellent enantioselectivity in up to $>99 \%$ ee was generally obtained with ester substituents on the C-C double bond (Table 2, entries 6 and 7). It was noteworthy that a slight drop in ee (only 98\%) with prolonged reaction time was observed as the ester substituents were replaced by ketones (Table 2, entries 8-10). The absolute configuration of Boc-protected product 3c was determined by X-ray crystallography (Figure 3). 
Table 1. Catalyst screening and optimization of cycloaddition reaction conditions ${ }^{a}$

\begin{tabular}{|c|c|c|c|c|c|c|}
\hline Entry & Catalyst & Solvent & Time (h) & Yield $(\%)^{b}$ & $\mathrm{dr}^{c}$ & ee $(\%)^{d}$ \\
\hline 1 & I & DCM & 6 & 43 & $1.1: 1$ & 32 \\
\hline 2 & II & DCM & 5 & 45 & $1.2: 1$ & 49 \\
\hline 3 & III & DCM & 5 & 61 & $2.0: 1$ & 89 \\
\hline 4 & IV & DCM & 2 & 67 & $3.2: 1$ & 95 \\
\hline 5 & $\mathbf{V}$ & DCM & 2 & 76 & $4.1: 1$ & 96 \\
\hline 6 & VI & DCM & 2 & 76 & $4.2: 1$ & 97 \\
\hline 7 & VII & DCM & 2 & 74 & 4.0:1 & 97 \\
\hline 8 & VI & toluene & 2 & 78 & $4.5: 1$ & $>99$ \\
\hline 9 & VI & $\mathrm{Et}_{2} \mathrm{O}$ & 4 & 63 & $3.8: 1$ & 98 \\
\hline $10^{e}$ & VI & toluene & 4 & 71 & $5.0: 1$ & 97 \\
\hline $11^{f}$ & VI & toluene & 5 & 78 & $5.6: 1$ & 32 \\
\hline $12^{g}$ & VI & toluene & 2 & 80 & $5.5: 1$ & $>99$ \\
\hline $13^{h}$ & VI & toluene & 4 & 75 & $5.2: 1$ & 89 \\
\hline
\end{tabular}

${ }^{a}$ All reactions were carried out by using isocyanide $\mathbf{1 a}(0.15 \mathrm{mmol}, 1.5$ equiv $)$ and methyleneindolinone $2 \mathbf{a}\left(0.1 \mathrm{mmol}, 1.0\right.$ equiv) with $10 \mathrm{~mol} \%$ of catalyst at $23{ }^{\circ} \mathrm{C} .{ }^{b}$ Isolated yield (major isomer). ${ }^{c} \mathrm{dr}$ determined by crude ${ }^{1} \mathrm{H}-\mathrm{NMR}$; the relative configuration of the minor diastereoisomer corresponds to that of compound 5a (see Table 3). ${ }^{d}$ ee was determined by chiral HPLC. ${ }^{e}$ Only 1.0 equivalent $1 \mathrm{a}$ was used. ${ }^{f}$ The reaction was conducted at $0{ }^{\circ} \mathrm{C} .{ }^{g} 5 \mathrm{~mol} \%$ catalyst was used. ${ }^{h}$ Catalyst loading was 2 mol\%. 


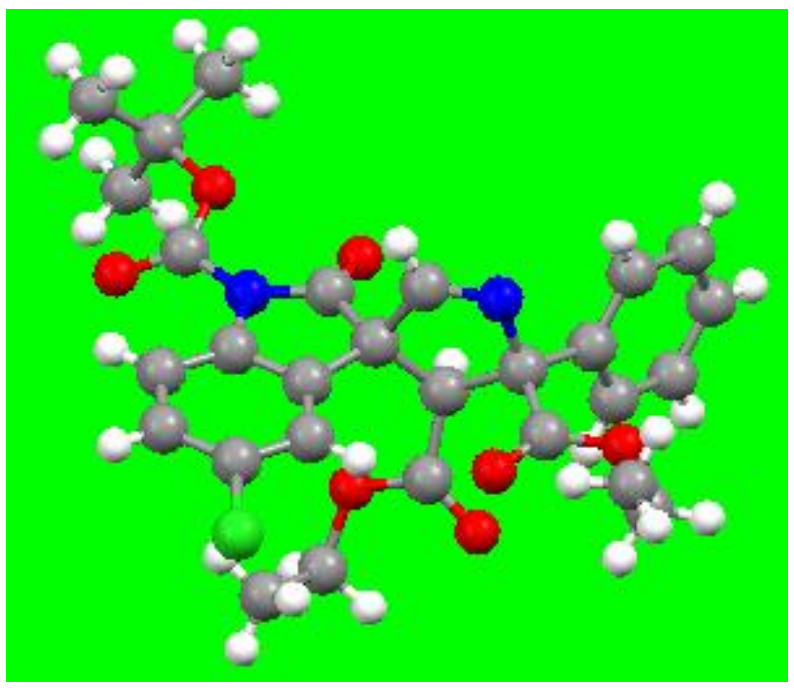

Figure 3. X-ray crystallography of Boc-protected product 3c.

In the course of cycloaddition between isocyanide and Boc-protected methyleneindolinones, besides the isolated major isomer, some minor compound was also observed in small amount. Since the spiro[indoline-3,3'-pyrrolidine] core is of promising biological properties, there is a high possibility that the minor stereoisomers process unique bioactivities. Therefore, it is a very great importance for "diversity-oriented synthesis", ${ }^{47}$ if the minor isomers could be formed as major products.

Based on the previous reports ${ }^{48}$ and our comprehension of this cycloaddition reaction, we anticipated that changing the electronic and steric properties of the methyleneindolinones by modification of the protecting group on nitrogen atom might be a promising approach to affect the diastereoselectivity. Remarkably, a new diastereoisomer ${ }^{49}$ was obtained as major product by simply replacing the protecting Boc-group with benzyl group. Several methyleneindolinones were selected for investigation of the generality of this approach (Table 3). Good yields and excellent enantioselectivities were obtained with methyleneindolinones bearing various substituents on the indolinone moiety and C-C double bond. Compared with the Boc-protected substrates, the reaction with the Bn-protecting group is generally slower and needs double catalyst loading (10 mol\%).

Although the electronic and steric properties of the substrates pay a crucial role to the stereoselectivity of cycloaddition reaction, from the results of this specific reaction, the steric hindrance of protecting group is the determinant factor for high diastereoselectivity. For further understanding the inherent insight, we obtained the structure of a Bn-protected substrate by Xray crystallography (see the Supporting material). As one face may be blocked by the presence of protecting group, leaving one site open for the cycloaddition. Thus, a catalytic activation mode of the cycloaddtion reactions was proposed (Figure 4). 
Table 2. Boc-protected substrates scope of the cycloaddition reactions ${ }^{a}$

$c$
$1 \mathbf{a}$

a All reactions were carried out by using isocyanide $1 \mathrm{a}(0.15 \mathrm{mmol}, 1.5$ equiv) and methyleneindolinone $\mathbf{2 a - 2 J}\left(0.1 \mathrm{mmol}, 1\right.$ equiv) with $5 \mathrm{~mol} \%$ of catalyst $\mathbf{V I}$ at $23{ }^{\circ} \mathrm{C} .{ }^{b}$ Isolated yield (major isomer). ${ }^{c} \mathrm{dr}$ determined by crude ${ }^{1} \mathrm{H}-\mathrm{NMR} .{ }^{d}$ ee was determined by chiral HPLC.

Further exploration of substrate scope was focused on the variation of isocyanides. Two more the methyl and benzyl isocyano-esters (1b and 1c) were tested as typical examples (Scheme 2). Gratifyingly, both reactions proceeded smoothly in high yield and enantioselectivity, further illustrating the validity and generality of this direct asymmetric cycloaddition.<smiles>[R]C(=O)C([N+]#N)c1ccccc1</smiles>

$1 \mathrm{~b}: \mathrm{R}=\mathrm{OMe}$, 1c: $\mathrm{R}=\mathrm{OBn}$,

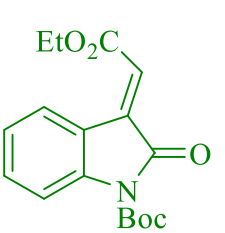

$\mathbf{2 a}$

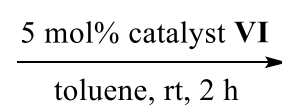

3k: $\mathrm{R}=\mathrm{OMe}, 71 \%$ yield, $5.0: 1 \mathrm{dr},>99 \%$ ee 3I: $\mathrm{R}=\mathrm{OBn}, 73 \%$ yield, $5.5: 1 \mathrm{dr},>99 \%$ ee

Scheme 2. Reactions with various isocyanides. 
Table 3. Scope of the [3+2] cycloaddition with Bn-protected substrates ${ }^{a}$

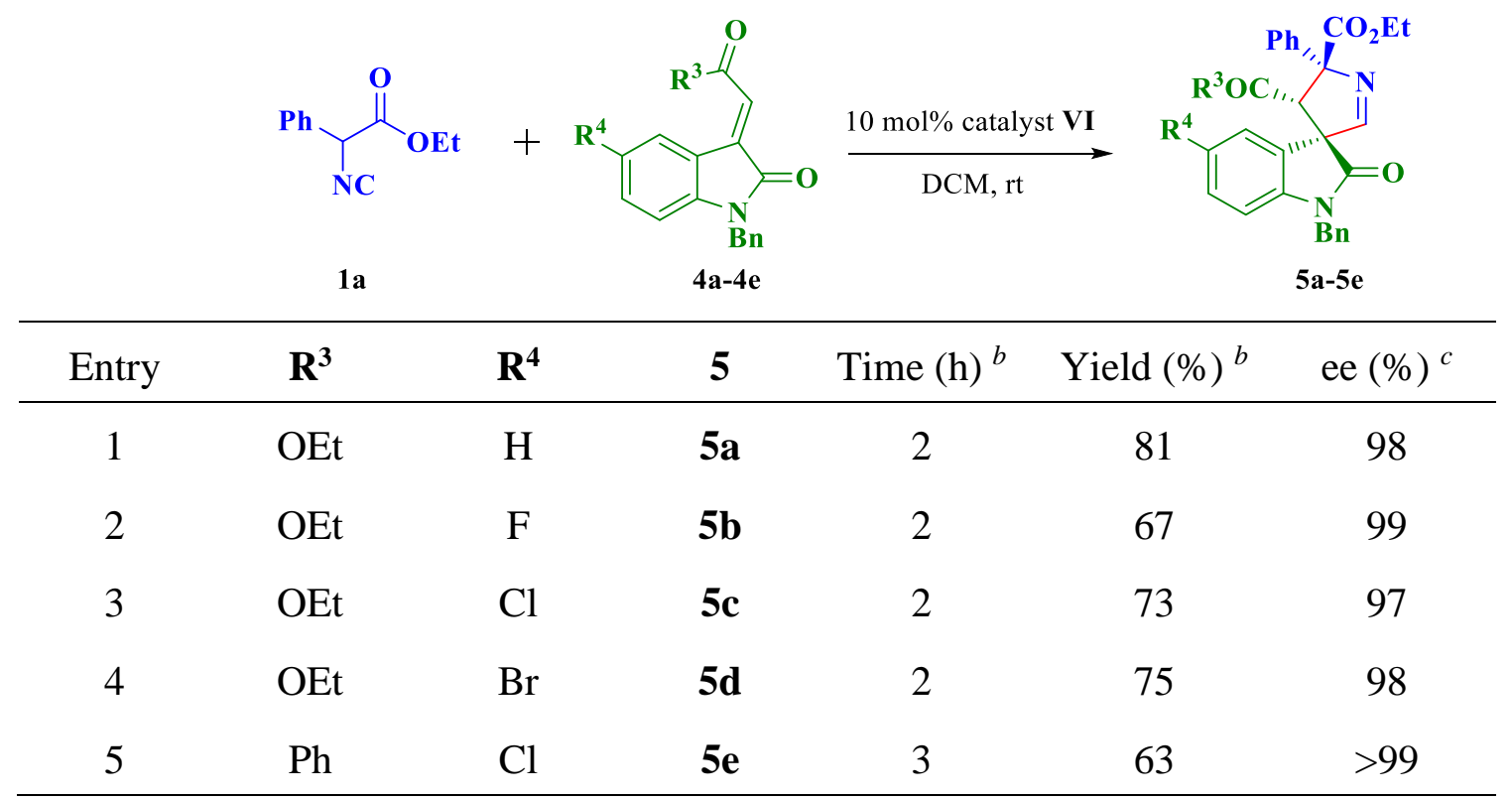

${ }^{a}$ All reactions were carried out by using isocyanide $\mathbf{1 a}(0.15 \mathrm{mmol}, 1.5$ equiv $)$ and methyleneindolinone $4 \mathbf{a}-4 \mathrm{e}(0.1 \mathrm{mmol}, 1$ equiv $)$ with $10 \mathrm{~mol} \%$ of catalyst $\mathbf{V I}$ at $23{ }^{\circ} \mathrm{C}$. ${ }^{b}$ Isolated yield. (Crude ${ }^{1} \mathrm{H}-\mathrm{NMR}$ displayed only one major isomer. However, the product is not very stable, which decreased the isolated yield.) ${ }^{c}$ ee was determined by chiral HPLC.

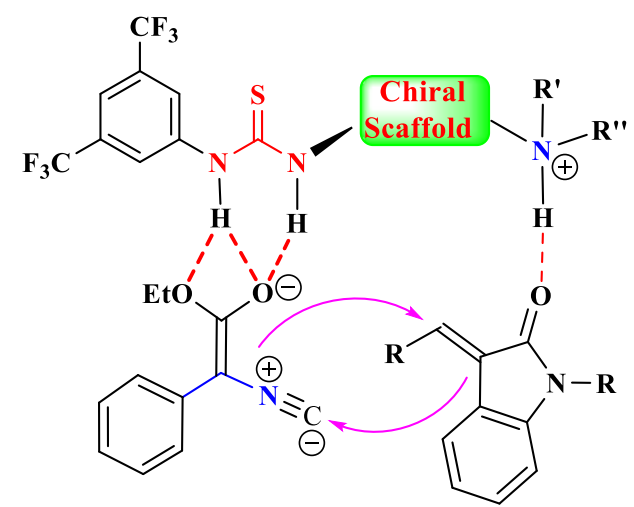

Figure 4. Proposed activation mode of the catalyst and substrates.

\section{Conclusions}

An asymmetric organocatalytic [3+2] cycloaddition of isocyanide and methyleneindolinone has been developed in good yield and excellent enantioselectivity, tolerating a broad range of substrates. The approach was associated with the formation of two quaternary and one tertiary carbon stereogenic centers, providing a highly stereoselective solution to the complex spiro[pyrrolilin-3,3'-oxindole] ring skeleton. Remarkably, two different major diastereoisomers 
can be selectively achieved by choosing different protecting groups on the nitrogen atom of methyleneindolinones. The success of this strategy opens up new perspectives in the construction of complex spiro[pyrrolidin-3,3'-oxindole] structure for a rapid access to biologically and pharmaceutically active candidates.

\section{Experimental Section}

General. Analytical thin layer chromatography (TLC) was performed using Merck 60 F254 precoated silica gel plate $(0.2 \mathrm{~mm}$ thickness). Subsequent to elution, plates were visualized using UV radiation $(254 \mathrm{~nm})$ on Spectroline Model ENF-24061/F at $254 \mathrm{~nm}$. Further visualization was possible by staining with basic solution of potassium permanganate or acidic solution of ceric molybdate. Flash chromatography was performed using Merck silica gel 60 with freshly distilled solvents. Columns were typically packed as slurry and equilibrated with the appropriate solvent system prior to use.

Proton nuclear magnetic resonance spectra ( ${ }^{1} \mathrm{H}$ NMR) were recorded on Bruker AMX 400 spectrophotometer $\left(\mathrm{CDCl}_{3}\right.$ as solvent). Chemical shifts for ${ }^{1} \mathrm{H}$ NMR spectra are reported as $\delta$ in units of parts per million (ppm) downfield from $\mathrm{SiMe}_{4}(\delta 0.0)$ and relative to the signal of chloroform-d ( $\delta 7.26$, singlet). Multiplicities were given as: s (singlet), $\mathrm{d}$ (doublet), $\mathrm{t}$ (triplet), dd (double of doublet) or $\mathrm{m}$ (multiplets). The number of protons (n) for a given resonance is indicated by $\mathrm{nH}$. Coupling constants are reported as a $\mathbf{J}$ value in Hz. Carbon nuclear magnetic resonance spectra $\left({ }^{13} \mathrm{C}\right.$ NMR) are reported as $\delta$ in units of parts per million (ppm) downfield from $\operatorname{SiMe}_{4}(\delta 0.0)$ and relative to the signal of chloroform-d $(\delta 77.0$, triplet).

Enantioselectivities were determined by High performance liquid chromatography (HPLC) analysis employing a Daicel Chiralpak AD-H or OD-H. Optical rotations were measured in $\mathrm{CH}_{2} \mathrm{Cl}_{2}$ on a Schmidt + Haensdch polarimeter (Polartronic MH8) with a $1.0 \mathrm{~mL}$ cell (c given in $\mathrm{g} / 100 \mathrm{~mL}$ ). Absolute configuration of the products was determined by X-ray.

High resolution mass spectrometry (HRMS) was recorded on Finnigan MAT 95×P spectrometer.

General experimental procedure for the construction of spirocyclic oxindoles with organocatalytic $[3+2]$ cycloaddition reactions $(3 \mathrm{a}-3 \mathrm{3J})$. To a solution of methyleneindolinone ( $0.1 \mathrm{mmol}, 1$ equiv), and isocyanide $(0.15 \mathrm{mmol}, 1.5$ equiv) in toluene $(0.2 \mathrm{~mL})$ was added catalyst VI ( $0.005 \mathrm{mmol}, 0.05$ equiv). The resulting mixture was stirred at room temperature ( 23 $\left.{ }^{\circ} \mathrm{C}\right)$. After the reaction completed, the mixture was quenched with water $(5 \mathrm{~mL})$ and extracted with ethyl acetate $(2 \times 5 \mathrm{~mL})$. The combined organic layer was washed with brine and dried over $\mathrm{Na}_{2} \mathrm{SO}_{4}$. The solvent was then removed under reduced pressure. The product was afforded by silica gel flash chromatography using gradient elution (EtOAc/Hexane $=1: 10$ to 1:6). 


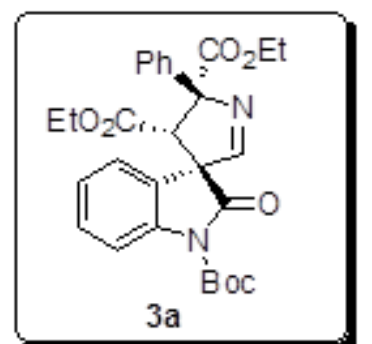

Chemical Formula: $\mathrm{C}_{28} \mathrm{H}_{30} \mathrm{~N}_{2} \mathrm{O}_{7}$ Exact Mass: 506.2053
1-tert-Butyl $\quad 4^{\prime}, 5^{\prime}$-diethyl $\quad\left(3 R, 4^{\prime} S, 5^{\prime} R\right)-2-0 x 0-5 '$-phenyl-4',5'dihydrospiro[indoline-3,3'-pyrrole]-1,4',5'-tricarboxylate $\quad(3 a) .{ }^{1} \mathrm{H}$ NMR (400 MHz, $\left.\mathrm{CDCl}_{3}\right) \delta 7.93(\mathrm{~d}, J 8.0 \mathrm{~Hz}, 1 \mathrm{H}), 7.78(\mathrm{~d}, J 8.0 \mathrm{~Hz}, 1 \mathrm{H})$, $7.57(\mathrm{~d}, J 7.2 \mathrm{~Hz}, 1 \mathrm{H}), 7.41-7.26(\mathrm{~m}, 5 \mathrm{H}), 7.20(\mathrm{t}, J 7.6 \mathrm{~Hz}, 1 \mathrm{H}), 4.35-4.22$ $(\mathrm{m}, 2 \mathrm{H}), 4.18(\mathrm{~s}, 1 \mathrm{H}), 3.88-3.77(\mathrm{~m}, 2 \mathrm{H}), 1.62(\mathrm{~s}, 9 \mathrm{H}), 1.26(\mathrm{t}, J 6.8 \mathrm{~Hz}$, $3 \mathrm{H}), 0.75(\mathrm{t}, J 7.2 \mathrm{~Hz}, 3 \mathrm{H}) .{ }^{13} \mathrm{C}-\mathrm{NMR}\left(100 \mathrm{MHz}, \mathrm{CDCl}_{3}\right) \delta 173.21$, $170.73,168.06,161.85,148.79$, 141.91, 139.87, 129.71, 128.12, 127.68, $126.83,126.58,125.00,123.97,114.90,87.89,85.08,70.27,62.69,62.50$, 61.15, 28.02, 13.90, 13.36. HPLC: Chiralpak AD-H (hexane/i-PrOH = 92/8, flow rate $1 \mathrm{~mL} / \mathrm{min}$, $\lambda=210 \mathrm{~nm}), \mathrm{t}_{\mathrm{R}}$ (major) $=6.7 \mathrm{~min}, \mathrm{t}_{\mathrm{R}}$ (minor) $=9.8 \mathrm{~min} ;>99 \%$ ee. $[\alpha]_{\mathrm{D}}{ }^{21}=-9.7(\mathrm{c}=1.0$, $\mathrm{CH}_{2} \mathrm{Cl}_{2}$ ). HRMS (ESI) calcd for $\mathrm{C}_{28} \mathrm{H}_{31} \mathrm{~N}_{2} \mathrm{O}_{7}(\mathrm{M}+\mathrm{H})^{+}, \mathrm{m} / \mathrm{z}$ 507.2131, found 507.2135.

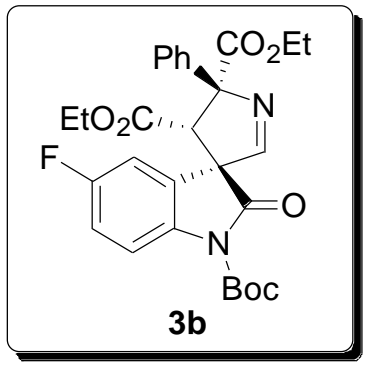

Chemical Formula: $\mathrm{C}_{28} \mathrm{H}_{29} \mathrm{FN}_{2} \mathrm{O}_{7}$ Exact Mass: 524.1959

1-tert-Butyl 4',5'-diethyl 5-fluoro $\left(3 R, 4^{\prime} S, 5 ' R\right)-2$-oxo-5'-phenyl-4',5'dihydrospiro [indoline- $3,3^{\prime}$-pyrrole]-1,4',5'-tricarboxylate $(3 \mathrm{~b}) .{ }^{1} \mathrm{H}$ NMR (400 MHz, $\left.\mathrm{CDCl}_{3}\right) \delta 7.96(\mathrm{dd}, J$ 4.4, $9.2 \mathrm{~Hz}, 1 \mathrm{H}), 7.67(\mathrm{dd}, J$ 2.8, $8.4 \mathrm{~Hz}, 1 \mathrm{H}), 7.58(\mathrm{~d}, J 7.2 \mathrm{~Hz}, 2 \mathrm{H}), 7.35(\mathrm{~m}, 4 \mathrm{H}), 7.12(\mathrm{~m}, 1 \mathrm{H}), 4.38-4.27$ $(\mathrm{m}, 2 \mathrm{H}), 4.19(\mathrm{~s}, 1 \mathrm{H}), 3.98-3.84(\mathrm{~m}, 2 \mathrm{H}), 1.63(\mathrm{~s}, 9 \mathrm{H}), 1.30(\mathrm{t}, J 7.2 \mathrm{~Hz}$, $3 \mathrm{H}), 0.84(\mathrm{t}, J 7.2 \mathrm{~Hz}, 3 \mathrm{H}) .{ }^{13} \mathrm{C}-\mathrm{NMR}\left(100 \mathrm{MHz}, \mathrm{CDCl}_{3}\right) \delta 172.86$, $170.58,167.83,161.41,161.28,158.85,148.73,141.62,135.86,128.16$, $127.77,126.80,125.90,125.81,116.32,116.19,116.09,114.46,114.20$, $88.07,85.30,70.22,62.70,62.65,61.31,28.00,13.90,13.44$. HPLC: Chiralpak OD-H (hexane/i-PrOH $=97 / 3$, flow rate $1 \mathrm{~mL} / \mathrm{min}, \lambda=210$ $\mathrm{nm}), \mathrm{t}_{\mathrm{R}}($ minor $)=7.4 \mathrm{~min}, \mathrm{t}_{\mathrm{R}}($ major $)=9.1 \mathrm{~min} ;>99 \%$ ee. $[\alpha]_{\mathrm{D}}^{21}=-16.4\left(\mathrm{c}=1.0, \mathrm{CH}_{2} \mathrm{Cl}_{2}\right)$. HRMS (ESI) calcd for $\mathrm{C}_{28} \mathrm{H}_{30} \mathrm{FN}_{2} \mathrm{O}_{7}(\mathrm{M}+\mathrm{H})^{+}, \mathrm{m} / \mathrm{z}$ 525.2037, found 525.2034.

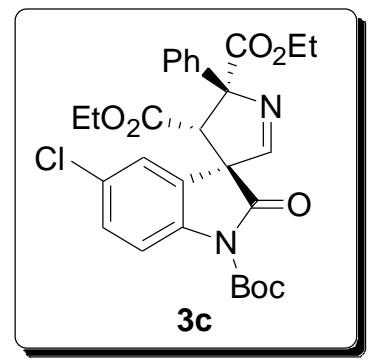

Chemical Formula: $\mathrm{C}_{28} \mathrm{H}_{29} \mathrm{CIN}_{2} \mathrm{O}_{7}$ Exact Mass: 540.1663

1-tert-Butyl 4',5'-diethyl 5-chloro $\left(3 R, 4^{\prime} S, 5 ' R\right)$-2-oxo-5'-phenyl-4',5'dihydrospiro [indoline- 3,3'-pyrrole]-1,4',5'-tricarboxylate $(3 \mathrm{c}) .{ }^{1} \mathrm{H}$ NMR (400 MHz, $\left.\mathrm{CDCl}_{3}\right) \delta 7.93(\mathrm{~d}, J 8.8 \mathrm{~Hz}, 1 \mathrm{H}), 7.84(\mathrm{~d}, J 2.0 \mathrm{~Hz}, 1 \mathrm{H})$, $7.59(\mathrm{~d}, J 7.2 \mathrm{~Hz}, 2 \mathrm{H}), 7.41-7.28(\mathrm{~m}, 5 \mathrm{H}), 4.39-4.29(\mathrm{~m}, 2 \mathrm{H}), 4.18(\mathrm{~s}$, $1 \mathrm{H}), 4.01-3.96(\mathrm{~m}, 1 \mathrm{H}), 3.88-3.84(\mathrm{~m}, 1 \mathrm{H}), 1.63(\mathrm{~s}, 9 \mathrm{H}), 1.32(\mathrm{t}, J 7.2 \mathrm{~Hz}$, $3 \mathrm{H}), 0.85(\mathrm{t}, J 7.1 \mathrm{~Hz}, 3 \mathrm{H}) .{ }^{13} \mathrm{C}-\mathrm{NMR}\left(100 \mathrm{MHz}, \mathrm{CDCl}_{3}\right) \delta 172.57$, $170.32,167.75,161.30,148.60,141.66,138.38,130.53,129.68,128.17$, $127.79,126.78,126.74,125.80,116.15,88.10,85.48,69.98,62.69$, 62.68, 61.35, 27.99, 13.96, 13.46. HPLC: Chiralpak AD-H (hexane/i$\mathrm{PrOH}=95 / 5$, flow rate $1 \mathrm{~mL} / \mathrm{min}, \lambda=210 \mathrm{~nm}), \mathrm{t}_{\mathrm{R}}($ minor $)=9.1 \mathrm{~min}, \mathrm{t}_{\mathrm{R}}$ (major) $=11.6 \mathrm{~min}$; $>99 \%$ ee. $[\alpha]_{\mathrm{D}}^{21}=39.0\left(\mathrm{c}=1.0, \mathrm{CH}_{2} \mathrm{Cl}_{2}\right)$. HRMS (ESI) calcd for $\mathrm{C}_{28} \mathrm{H}_{30} \mathrm{ClN}_{2} \mathrm{O}_{7}(\mathrm{M}+\mathrm{H})^{+}, \mathrm{m} / \mathrm{z} 541.1742$, found 541.1745. 


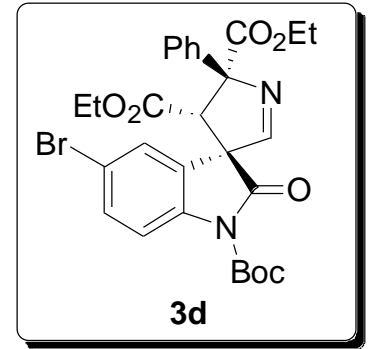

Chemical Formula: $\mathrm{C}_{28} \mathrm{H}_{29} \mathrm{BrN}_{2} \mathrm{O}_{7}$ Exact Mass: 584.1158

1-tert-Butyl 4',5'-diethyl 5-bromo $\left(3 R, 4^{\prime} S, 5^{\prime} R\right)$-2-oxo-5'-phenyl-4',5'dihydrospiro[indoline $\mathbf{- 3 , 3} \mathbf{3}^{\prime}$-pyrrole]-1,4',5'-tricarboxylate (3d). ${ }^{1} \mathrm{H}$ NMR (400 MHz, CDCl $) \delta 7.95(\mathrm{~s}, 1 \mathrm{H}), 7.88(\mathrm{~d}, J 8.8 \mathrm{~Hz}, 1 \mathrm{H}), 7.59-7.54$ $(\mathrm{m}, 3 \mathrm{H}), 7.39-7.28(\mathrm{~m}, 4 \mathrm{H}), 4.41-4.28(\mathrm{~m}, 2 \mathrm{H}), 4.17(\mathrm{~s}, 1 \mathrm{H}), 4.03-3.95$ (m, 1H), 3.90-3.82 (m, 1H), 1.63 (s, 9H), 1.33 (t, J $7.2 \mathrm{~Hz}, 3 \mathrm{H}), 0.85$ (t, $J$ $7.2 \mathrm{~Hz}, 3 \mathrm{H}) .{ }^{13} \mathrm{C}-\mathrm{NMR}\left(100 \mathrm{MHz}, \mathrm{CDCl}_{3}\right) \delta 172.44,170.24,167.73$, $161.29,148.58,141.66,138.89,132.62,129.51,128.18,127.80,126.78$, $126.12,117.99,116.55,88.11,85.52,69.88,62.70,62.69,61.37,27.99$, 14.01, 13.48. HPLC: Chiralpak AD-H (hexane/i-PrOH $=97 / 3$, flow rate 1 $\mathrm{mL} / \mathrm{min}, \lambda=210 \mathrm{~nm}), \mathrm{t}_{\mathrm{R}}($ minor $)=15.0 \mathrm{~min}, \mathrm{t}_{\mathrm{R}}($ major $)=21.0 \mathrm{~min}$; $>99 \%$ ee. $[\alpha]_{D}^{21}=39.2\left(\mathrm{c}=1.0, \mathrm{CH}_{2} \mathrm{Cl}_{2}\right)$. HRMS $(\mathrm{ESI})$ calcd for $\mathrm{C}_{28} \mathrm{H}_{30} \mathrm{BrN}_{2} \mathrm{O}_{7}(\mathrm{M}+\mathrm{H})^{+}, \mathrm{m} / \mathrm{z}$ 585.1236 , found 585.1234.

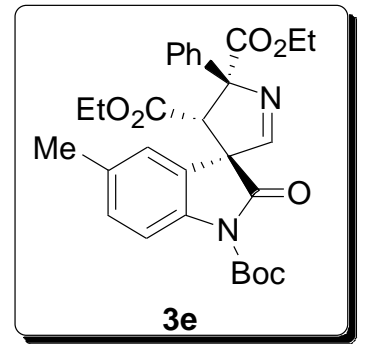

Chemical Formula: $\mathrm{C}_{29} \mathrm{H}_{32} \mathrm{~N}_{2} \mathrm{O}_{7}$ Exact Mass: 520.2210

1-tert-Butyl 4',5'-diethyl 5-methyl $\left(3 R, 44^{\prime} S, 5^{\prime} R\right)$-2-oxo-5'-phenyl-4',5'dihydro-spiro[indoline-3,3'-pyrrole]-1,4',5'-tricarboxylate $(3 e) .{ }^{1} \mathrm{H}$ NMR $\left(400 \mathrm{MHz}, \mathrm{CDCl}_{3}\right) \delta 7.82(\mathrm{~d}, J 8.4 \mathrm{~Hz}, 1 \mathrm{H}), 7.59-7.55(\mathrm{~m}, 3 \mathrm{H})$, 7.40-7.31 (m, 4H), $7.20(\mathrm{~d}, J 8.3 \mathrm{~Hz}, 1 \mathrm{H}), 4.37-4.28(\mathrm{~m}, 2 \mathrm{H}), 4.19(\mathrm{~s}, 1 \mathrm{H})$, 3.95-3.78 (m, 2H), $2.37(\mathrm{~s}, 3 \mathrm{H}), 1.63(\mathrm{~s}, 9 \mathrm{H}), 1.31(\mathrm{t}, J 7.2 \mathrm{~Hz}, 3 \mathrm{H}), 0.78$ (t, $J 7.2 \mathrm{~Hz}, 3 \mathrm{H}) .{ }^{13} \mathrm{C}-\mathrm{NMR}\left(100 \mathrm{MHz}, \mathrm{CDCl}_{3}\right) \delta 173.27,170.54,168.08$, $162.05,148.83,142.01,137.50,134.61,130.15,128.11,127.66,126.97$, $126.82,123.85,114.70,87.83,84.90,70.32,62.61,62.39,61.10,28.03$, 21.18, 13.97, 13.38. HPLC: Chiralpak AD-H (hexane/i-PrOH $=95 / 5$, flow rate $1 \mathrm{~mL} / \mathrm{min}, \lambda=210 \mathrm{~nm}$ ), $\mathrm{t}_{\mathrm{R}}($ minor $)=8.6 \mathrm{~min}, \mathrm{t}_{\mathrm{R}}$ (major) $=11.5 \mathrm{~min} ;>99 \%$ ee. $[\alpha]_{\mathrm{D}}{ }^{21}=15.1$ (c = 1.0, $\mathrm{CH}_{2} \mathrm{Cl}_{2}$ ). HRMS (ESI) calcd for $\mathrm{C}_{29} \mathrm{H}_{33} \mathrm{~N}_{2} \mathrm{O}_{7}(\mathrm{M}+\mathrm{H})^{+}, \mathrm{m} / \mathrm{z}$ 521.2288, found 521.2289.

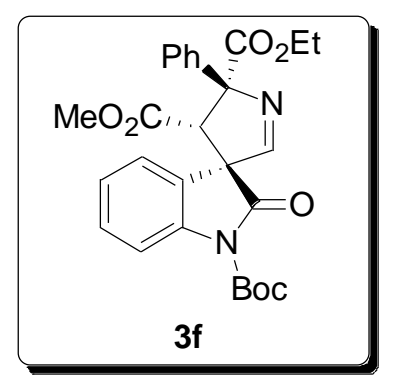

Chemical Formula: $\mathrm{C}_{27} \mathrm{H}_{28} \mathrm{~N}_{2} \mathrm{O}_{7}$

Exact Mass: 492.1897
1-tert-Butyl 5 '-ethyl $\quad 4^{\prime}$-methyl $\left(3 R, 4^{\prime} S, 5^{\prime} R\right)$-2-oxo-5'-phenyl-4',5'dihydrospiro[indoline- $3,3^{\prime}$-pyrrole]-1,4',5'-tricarboxylate $(3 \mathrm{f}) .{ }^{1} \mathrm{H}$ NMR $\left(400 \mathrm{MHz}, \mathrm{CDCl}_{3}\right) \delta 7.97(\mathrm{~d}, J 8.0 \mathrm{~Hz}, 1 \mathrm{H}), 7.71(\mathrm{~d}, J 7.6 \mathrm{~Hz}, 1 \mathrm{H})$, $7.59(\mathrm{~d}, J 1.2 \mathrm{~Hz}, 2 \mathrm{H}), 7.57-7.33(\mathrm{~m}, 5 \mathrm{H}), 7.23(\mathrm{t}, J 7.6 \mathrm{~Hz}, 1 \mathrm{H}), 4.37-$ $4.26(\mathrm{~m}, 2 \mathrm{H}), 4.22(\mathrm{~s}, 1 \mathrm{H}), 3.38(\mathrm{~s}, 3 \mathrm{H}), 1.64(\mathrm{~s}, 9 \mathrm{H}), 1.29(\mathrm{t}, J 7.2 \mathrm{~Hz}$, $3 \mathrm{H}) .{ }^{13} \mathrm{C}-\mathrm{NMR}\left(100 \mathrm{MHz}, \mathrm{CDCl}_{3}\right) \delta 172.94,170.76,168.65,161.89$, $148.79,141.71,139.63,129.75,128.17,127.76,126.80,126.37,124.92$, 123.72, 114.97, 88.24, 85.14, 70.25, 62.53, 62.31, 52.05, 28.03, 13.92. HPLC: Chiralpak AD-H (hexane/i-PrOH $=95 / 5$, flow rate $1 \mathrm{~mL} / \mathrm{min}, \lambda=$ $210 \mathrm{~nm}), \mathrm{t}_{\mathrm{R}}($ major $)=11.7 \mathrm{~min}, \mathrm{t}_{\mathrm{R}}($ minor $)=14.4 \mathrm{~min} ;>99 \% \mathrm{ee} \cdot[\alpha]_{\mathrm{D}}^{21}=$ $-7.5\left(\mathrm{c}=1.0, \mathrm{CH}_{2} \mathrm{Cl}_{2}\right.$ ). HRMS (ESI) calcd for $\mathrm{C}_{27} \mathrm{H}_{29} \mathrm{~N}_{2} \mathrm{O}_{7}(\mathrm{M}+\mathrm{H})^{+}, \mathrm{m} / \mathrm{z} 493.1975$, found 493.1969. 
4'-Benzyl 1-tert-butyl 5'-ethyl (3R,4'S,5'R)-2-oxo-5'-phenyl-4',5'-dihydrospiro [indoline-

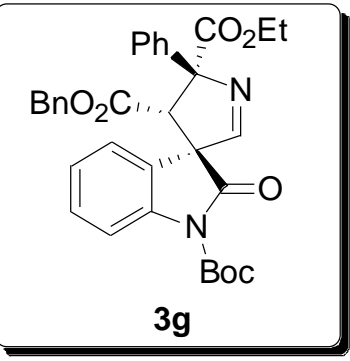

Chemical Formula: $\mathrm{C}_{33} \mathrm{H}_{32} \mathrm{~N}_{2} \mathrm{O}_{7}$

Exact Mass: 568.2210 3,3'-pyrrole]-1,4',5'-tricarboxylate (3g). ${ }^{1} \mathrm{H}-\mathrm{NMR}\left(400 \mathrm{MHz}, \mathrm{CDCl}_{3}\right) \delta$ $7.89(\mathrm{~d}, J 8.0 \mathrm{~Hz}, 1 \mathrm{H}), 7.78(\mathrm{~d}, J 7.6 \mathrm{~Hz}, 1 \mathrm{H}), 7.57(\mathrm{~d}, J 1.4 \mathrm{~Hz}, 2 \mathrm{H})$, 7.56-7.18 (m, 9H), $6.91(\mathrm{~d}, J 6.4 \mathrm{~Hz}, 2 \mathrm{H}), 4.87(\mathrm{~d}, J 12 \mathrm{~Hz}, 1 \mathrm{H}), 4.69$ (d, $J 12.0 \mathrm{~Hz}, 1 \mathrm{H}), 4.37-4.25(\mathrm{~m}, 3 \mathrm{H}), 1.60(\mathrm{~s}, 9 \mathrm{H}), 1.27$ (t, $J 7.2 \mathrm{~Hz}, 3 \mathrm{H})$. ${ }^{13} \mathrm{C}-\mathrm{NMR}\left(100 \mathrm{MHz}, \mathrm{CDCl}_{3}\right) \delta 173.02,170.68,167.95,161.98,148.62$, $141.76,139.76,134.39,129.77,128.41,128.37,128.30,128.14,127.72$, $126.85,126.47,125.05,123.68,115.09,88.01,84.95,70.24,67.35$, 62.54, 62.52, 27.99, 13.90. HPLC: Chiralpak AD-H (hexane/i-PrOH = $90 / 10$, flow rate $1 \mathrm{~mL} / \mathrm{min}, \lambda=210 \mathrm{~nm}), t_{R}$ (major) $=10.8 \mathrm{~min}, \mathrm{t}_{\mathrm{R}}($ minor) $=13.2 \mathrm{~min} ;>99 \%$ ee. $[\alpha]_{\mathrm{D}}^{21}=-9.7\left(\mathrm{c}=1.0, \mathrm{CH}_{2} \mathrm{Cl}_{2}\right)$. HRMS (ESI) calcd for $\mathrm{C}_{33} \mathrm{H}_{33} \mathrm{~N}_{2} \mathrm{O}_{7}(\mathrm{M}+\mathrm{H})^{+}, \mathrm{m} / \mathrm{z}$ 569.2288, found 569.2290.

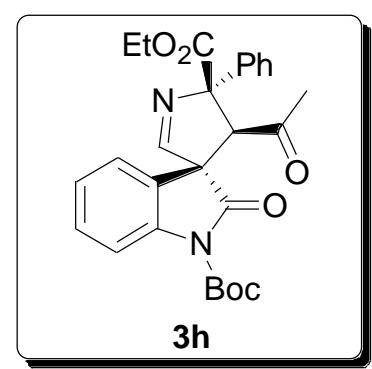

Chemical Formula: $\mathrm{C}_{27} \mathrm{H}_{28} \mathrm{~N}_{2} \mathrm{O}_{6}$ Exact Mass: 476.1947

\section{1-tert-Butyl $\quad 5^{\prime}$-ethyl $\quad 4^{\prime}$-acetyl $\quad\left(3 R, 4^{\prime} S, 5^{\prime} S\right)$-2-oxo-5'-phenyl-4',5'-} dihydrospiro[indoline-3,3'-pyrrole]-1,5'-dicarboxylate (3h). ${ }^{1} \mathrm{H}-\mathrm{NMR}$ $\left(400 \mathrm{MHz}, \mathrm{CDCl}_{3}\right) \delta 7.99(\mathrm{~d}, J 8.0 \mathrm{~Hz}, 1 \mathrm{H}), 7.66(\mathrm{~d}, J 7.6 \mathrm{~Hz}, 1 \mathrm{H}), 7.50$ $7.34(\mathrm{~m}, 6 \mathrm{H}), 7.24-7.21(\mathrm{~m}, 2 \mathrm{H}), 4.42-4.28(\mathrm{~m}, 2 \mathrm{H}), 4.23(\mathrm{~s}, 1 \mathrm{H}), 1.73(\mathrm{~s}$, $3 \mathrm{H}), 1.65(\mathrm{~s}, 9 \mathrm{H}), 1.33(\mathrm{t}, J 7.2 \mathrm{~Hz}, 3 \mathrm{H}) .{ }^{13} \mathrm{C}-\mathrm{NMR}\left(100 \mathrm{MHz}, \mathrm{CDCl}_{3}\right) \delta$ 201.64, 173.05, 171.16, 161.31, 148.60, 141.67, 139.48, 130.08, 128.28, $127.80,126.74,126.59,125.45,123.18,115.29,87.98,85.45,71.13$, 69.56, 62.52, 30.20, 28.03, 13.91. HPLC: Chiralpak OD-H (hexane/i$\mathrm{PrOH}=97 / 3$, flow rate $1 \mathrm{~mL} / \mathrm{min}, \lambda=210 \mathrm{~nm}), \mathrm{t}_{\mathrm{R}}($ major $)=17.9 \mathrm{~min}, \mathrm{t}_{\mathrm{R}}$ $($ minor $)=21.6 \mathrm{~min} ;>99 \%$ ee. $[\alpha]_{\mathrm{D}}^{21}=35.2\left(\mathrm{c}=1.0, \mathrm{CH}_{2} \mathrm{Cl}_{2}\right)$. HRMS (ESI) calcd for $\mathrm{C}_{27} \mathrm{H}_{29} \mathrm{~N}_{2} \mathrm{O}_{6}(\mathrm{M}+\mathrm{H})^{+}, \mathrm{m} / \mathrm{z}$ 477.2026, found 477.2028.

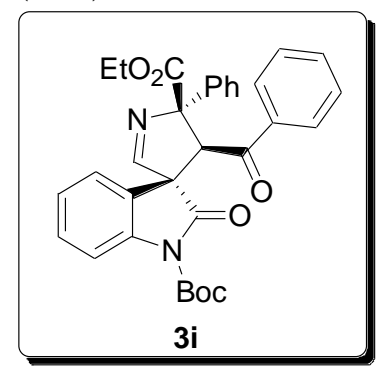

Chemical Formula: $\mathrm{C}_{32} \mathrm{H}_{30} \mathrm{~N}_{2} \mathrm{O}_{6}$ Exact Mass: 538.2104

1-tert-Butyl 5'-ethyl 4'-benzoyl $\left(3 R, 4^{\prime} S, 5^{\prime} S\right)$-2-oxo-5'-phenyl-4',5'dihydrospiro[indoline-3,3'-pyrrole]-1,5'-dicarboxylate (3i). ${ }^{1} \mathrm{H}-\mathrm{NMR}$ $\left(400 \mathrm{MHz}, \mathrm{CDCl}_{3}\right) \delta 7.72(\mathrm{~d}, J 7.6 \mathrm{~Hz}, 1 \mathrm{H}), 7.56(\mathrm{~d}, J 7.2 \mathrm{~Hz}, 2 \mathrm{H}), 7.49$ $(\mathrm{d}, \mathrm{J} 8.0 \mathrm{~Hz}, 1 \mathrm{H}), 7.42-7.28(\mathrm{~m}, 7 \mathrm{H}), 7.21-7.12(\mathrm{~m}, 4 \mathrm{H}), 5.05(\mathrm{~s}, 1 \mathrm{H})$, 4.48-4.30 (m, 2H), $1.61(\mathrm{~s}, 9 \mathrm{H}), 1.32(\mathrm{t}, J 7.2 \mathrm{~Hz}, 3 \mathrm{H}) .{ }^{13} \mathrm{C}-\mathrm{NMR}(100$ $\left.\mathrm{MHz}, \mathrm{CDCl}_{3}\right) \delta 196.40,173.54,171.15,160.73,148.23,142.43,139.16$, $137.37,133.00,129.79,128.33,128.19,127.59,127.28,127.22,126.93$, 125.07, 122.31, 114.39, 87.91, 84.96, 70.66, 66.77, 62.48, 28.01, 13.88. HPLC: Chiralpak IC (hexane/i-PrOH $=90 / 10$, flow rate $1 \mathrm{~mL} / \mathrm{min}, \lambda=$ $210 \mathrm{~nm}), \mathrm{t}_{\mathrm{R}}($ major $)=13.8 \mathrm{~min}, \mathrm{t}_{\mathrm{R}}($ minor $)=29.5 \mathrm{~min} ; 97 \%$ ee. $[\alpha]_{\mathrm{D}}^{21}=-$

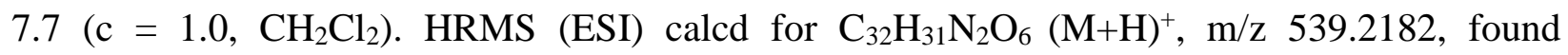
539.2183. 


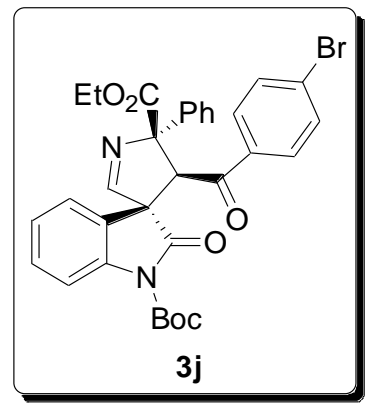

Chemical Formula: $\mathrm{C}_{32} \mathrm{H}_{29} \mathrm{BrN}_{2} \mathrm{O}_{6}$ Exact Mass: 616.1209

1-tert-Butyl 5'-ethyl $\quad 4^{\prime}$-(4-bromobenzoyl) $\quad\left(3 R, 4^{\prime} S, 5^{\prime} S\right)-2-0 x 0-5 '-$ phenyl-4',5'-dihydrospiro- [indoline-3,3'-pyrrole]-1,5'-dicarboxylate (3J). ${ }^{1} \mathrm{H}-\mathrm{NMR}\left(400 \mathrm{MHz}, \mathrm{CDCl}_{3}\right) \delta 7.67(\mathrm{~d}, J 7.6 \mathrm{~Hz}, 1 \mathrm{H}), 7.53(\mathrm{~d}, J 8.0$ $\mathrm{Hz}, 3 \mathrm{H}), 7.39-7.33$ (m, 6H), 7.26-7.23 (m, 1H), 7.19-7.13 (m, 3H), 4.99 $(\mathrm{s}, 1 \mathrm{H}), 4.44-4.31(\mathrm{~m}, 2 \mathrm{H}), 1.63(\mathrm{~s}, 9 \mathrm{H}), 1.31(\mathrm{t}, J 7.2 \mathrm{~Hz}, 3 \mathrm{H}) .{ }^{13} \mathrm{C}-\mathrm{NMR}$ $\left(100 \mathrm{MHz}, \mathrm{CDCl}_{3}\right) \delta 195.40,173.45,170.98,160.61,148.05,142.22$, $139.11,136.10,131.63,129.99,128.80,128.26,128.19,127.70,127.78$, 126.87, 125.14, 122.15, 114.47, 87.95, 85.26, 70.58, 66.65, 62.53, 28.02, 13.87. HPLC: Chiralpak AD-H (hexane/i-PrOH $=85 / 15$, flow rate 1 $\mathrm{mL} / \mathrm{min}, \lambda=210 \mathrm{~nm}), \mathrm{t}_{\mathrm{R}}($ major $)=16.9 \mathrm{~min}, \mathrm{t}_{\mathrm{R}}($ minor $)=26.5 \mathrm{~min} ; 98 \%$ ee. $[\alpha]_{\mathrm{D}}{ }^{21}=-15.9\left(\mathrm{c}=1.0, \mathrm{CH}_{2} \mathrm{Cl}_{2}\right)$. HRMS (ESI) calcd for $\mathrm{C}_{32} \mathrm{H}_{30} \mathrm{BrN}_{2} \mathrm{O}_{6}(\mathrm{M}+\mathrm{H})^{+}, \mathrm{m} / \mathrm{z}$ 617.1287, found 617.1284 .

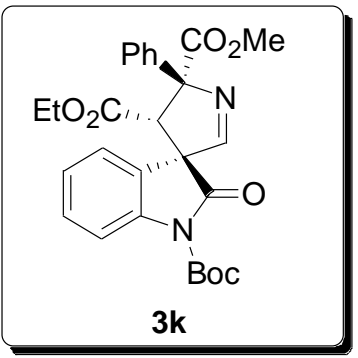

Chemical Formula:

$\mathrm{C}_{27} \mathrm{H}_{28} \mathrm{~N}_{2} \mathrm{O}_{7}$ Exact Mass: 492.1897

1-tert-Butyl $\quad 4^{\prime}$-ethyl $\quad 5^{\prime}$-methyl $\quad\left(3 R, 4^{\prime} S, 5^{\prime} S\right)-2$-oxo-5'-phenyl-4',5'dihydrospiro [indoline-3,3'- pyrrole]-1,4',5'-tricarboxylate (3k). ${ }^{1} \mathrm{H}$ NMR (400 MHz, CDCl $\left.{ }_{3}\right) \delta 7.93(\mathrm{~d}, \mathrm{~J} 8.0 \mathrm{~Hz}, 1 \mathrm{H}), 7.77$ (d, J 7.6 Hz, 1H), $7.57(\mathrm{~d}, J 7.2 \mathrm{~Hz}, 2 \mathrm{H}), 7.41-7.30(\mathrm{~m}, 5 \mathrm{H}), 7.21(\mathrm{t}, J 7.6 \mathrm{~Hz}, 1 \mathrm{H}), 4.20(\mathrm{~s}$, $1 \mathrm{H}), 3.87-3.76(\mathrm{~m}, 5 \mathrm{H})(-\mathrm{CH} 3$ and $-\mathrm{CH} 2$ overlap), $1.62(\mathrm{~s}, 9 \mathrm{H}), 0.73(\mathrm{t}, J$ $7.2 \mathrm{~Hz}, 3 \mathrm{H}) .{ }^{13} \mathrm{C}-\mathrm{NMR}\left(100 \mathrm{MHz}, \mathrm{CDCl}_{3}\right) \delta 173.15,171.33,168.13$, $161.88,148.77,141.75,139.91,129.78,128.22,127.80,126.84,126.53$, $125.10,123.82,114.91,87.70,85.11,70.38,62.78,61.25,53.34,28.02$, 13.35. HPLC: Chiralpak AD-H (hexane/i-PrOH $=85 / 15$, flow rate 1 $\mathrm{mL} / \mathrm{min}, \lambda=210 \mathrm{~nm}), \mathrm{t}_{\mathrm{R}}$ (major) $=6.7 \mathrm{~min}, \mathrm{t}_{\mathrm{R}}($ minor $)=8.4 \mathrm{~min} ;>99 \%$ ee. $[\alpha]_{\mathrm{D}}{ }^{21}=-12.8\left(\mathrm{c}=1.2, \mathrm{CH}_{2} \mathrm{Cl}_{2}\right)$. HRMS (ESI) calcd for $\mathrm{C}_{27} \mathrm{H}_{29} \mathrm{~N}_{2} \mathrm{O}_{7}(\mathrm{M}+\mathrm{H})^{+}, \mathrm{m} / \mathrm{z}$ 493.1975, found 493.1972 .

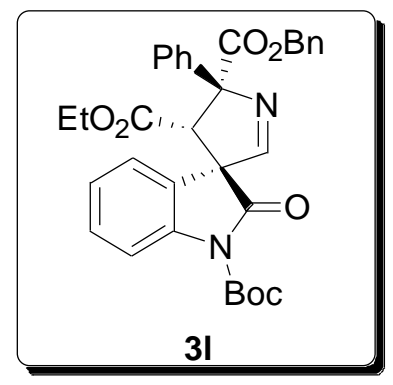

Chemical Formula: $\mathrm{C}_{33} \mathrm{H}_{32} \mathrm{~N}_{2} \mathrm{O}_{7}$ Exact Mass: 568.2210

-5'-Benzyl 1-tert-butyl $4^{\prime}$-ethyl $\left(3 R, 4^{\prime} S, 5^{\prime} S\right)$-2-oxo-5'-phenyl-4',5'dihydrospiro [indoline-3,3'- pyrrole]-1,4',5'-tricarboxylate $(3 \mathrm{l}) .{ }^{1} \mathrm{H}$ NMR (400 MHz, $\left.\mathrm{CDCl}_{3}\right) \delta 7.91(\mathrm{~d}, J 8.0 \mathrm{~Hz}, 1 \mathrm{H}), 7.58-7.54(\mathrm{~m}, 3 \mathrm{H})$, 7.38-7.29 (m, 7H), 7.26-7.22 (m, 3H), 7.02 (t, $J 7.6 \mathrm{~Hz}, 1 \mathrm{H}), 5.27$ (dd, $J$ 12.6, $24.5 \mathrm{~Hz}, 2 \mathrm{H}), 4.22(\mathrm{~s}, 1 \mathrm{H}), 3.84-3.72(\mathrm{~m}, 2 \mathrm{H}), 1.61(\mathrm{~s}, 9 \mathrm{H}), 0.70(\mathrm{t}$, J $7.2 \mathrm{~Hz}, 3 \mathrm{H}) .{ }^{13} \mathrm{C}-\mathrm{NMR}\left(100 \mathrm{MHz}, \mathrm{CDCl}_{3}\right) \delta 173.14,170.43,168.01$, $162.00,148.77,141.78,139.82,135.25,129.68,128.42,128.17,128.11$, $127.94,127.79,126.88,126.52,124.97,123.74,114.88,87.82,85.10$, 70.32, 67.94, 62.63, 61.22, 28.02, 13.32. HPLC: Chiralpak AD-H (hexane/i-PrOH $=90 / 10$, flow rate $1 \mathrm{~mL} / \mathrm{min}, \lambda=210 \mathrm{~nm}), \mathrm{t}_{\mathrm{R}}($ minor $)=$ $14.3 \mathrm{~min}, \mathrm{t}_{\mathrm{R}}$ (major) $=19.4 \mathrm{~min} ;>99 \%$ ee. $[\alpha]_{\mathrm{D}}{ }^{21}=-13.4\left(\mathrm{c}=0.8, \mathrm{CH}_{2} \mathrm{Cl}_{2}\right)$. HRMS $(\mathrm{ESI})$ calcd for $\mathrm{C}_{33} \mathrm{H}_{33} \mathrm{~N}_{2} \mathrm{O}_{7}(\mathrm{M}+\mathrm{H})^{+}, \mathrm{m} / \mathrm{z}$ 569.2288, found 569.2290.

General procedure for the construction of spirocyclic oxindoles with organocatalytic [3+2]cycloaddition reactions (5a-3f): To a solution of methyleneindolinone $(0.1 \mathrm{mmol}, 1$ equiv) and isocyanide $1 \mathbf{a}(0.15 \mathrm{mmol}, 1.5$ equiv $)$ in DCM $(0.2 \mathrm{~mL})$ was added catalyst VI $(0.01$ 
mmol, 0.1 equiv). The resulting mixture was stirred at room temperature $\left(23{ }^{\circ} \mathrm{C}\right)$. After the reaction completed, the mixture was quenched with water $(5 \mathrm{~mL})$ and extracted with DCM $(2 \times 5$ $\mathrm{mL}$ ). The combined organic layer was washed with brine and dried over $\mathrm{Na}_{2} \mathrm{SO}_{4}$. The solvent was then removed under reduced pressure. The product was afforded by silica gel flash chromatography using gradient elution (EtOAc/Hexane $=1: 10$ to $1: 6$ ).

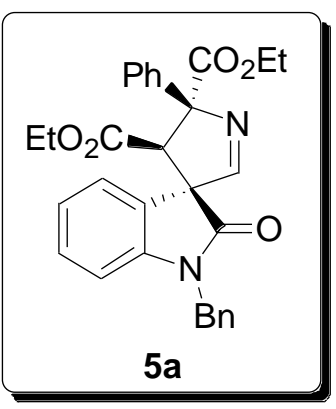

Chemical Formula: $\mathrm{C}_{30} \mathrm{H}_{28} \mathrm{~N}_{2} \mathrm{O}_{5}$

Exact Mass: 496.1998

Diethyl 1-benzyl (3R,4'S,5'S)-2-oxo-5'-phenyl-4',5'dihydrospiro[indoline-3,3'-pyrrole]-4',5'- dicarboxylate (5a). ${ }^{1} \mathrm{H}-\mathrm{NMR}$ $\left(400 \mathrm{MHz}, \mathrm{CDCl}_{3}\right) \delta 7.73(\mathrm{~d}, J 1.2 \mathrm{~Hz}, 2 \mathrm{H}), 7.71(\mathrm{~s}, 1 \mathrm{H}), 7.56-7.32(\mathrm{~m}$, $8 \mathrm{H}), 7.26(\mathrm{t}, J 7.6 \mathrm{~Hz}, 1 \mathrm{H}), 7.17(\mathrm{~d}, \mathrm{~J} 7.6 \mathrm{~Hz}, 1 \mathrm{H}), 6.98(\mathrm{t}, 7.6 \mathrm{~Hz}, 1 \mathrm{H})$, $6.80(\mathrm{~d}, \mathrm{~J} 8.0 \mathrm{~Hz}, 1 \mathrm{H}), 5.03(\mathrm{~d}, J$ 15.6, Hz, 1H), 4.96 (d, J 15.6, Hz, $1 \mathrm{H}), 4.86(\mathrm{~s}, 1 \mathrm{H}), 4.36(\mathrm{q}, J 7.1 \mathrm{~Hz}, 2 \mathrm{H}), 3.67-3.63(\mathrm{~m}, 2 \mathrm{H}), 1.32(\mathrm{t}, J 7.2$ $\mathrm{Hz}, 3 \mathrm{H}), 0.78(\mathrm{t}, J 7.2 \mathrm{~Hz}, 3 \mathrm{H}) .{ }^{13} \mathrm{C}-\mathrm{NMR}\left(100 \mathrm{MHz}, \mathrm{CDCl}_{3}\right) \delta 173.41$, $171.56,167.83$, 163.22, 143.64, 138.95, 135.18, 129.88, 128.96, 128.19, $128.09,127.93,127.34,126.81,126.64,123.80,122.98,109.59,89.39$, $68.51,62.50,60.66,59.18,44.38,14.02,13.49$. HPLC: Chiralpak IB (hexane/i-PrOH $=85 / 15$, flow rate $1 \mathrm{~mL} / \mathrm{min}, \lambda=210 \mathrm{~nm}), \mathrm{t}_{\mathrm{R}}($ minor) $=$ $10.9 \mathrm{~min}, \mathrm{t}_{\mathrm{R}}($ major$)=12.6 \mathrm{~min} ; 98 \%$ ee. $[\alpha]_{\mathrm{D}}{ }^{21}=58.7\left(\mathrm{c}=1.0, \mathrm{CH}_{2} \mathrm{Cl}_{2}\right)$. HRMS $(\mathrm{ESI})$ calcd for $\mathrm{C}_{30} \mathrm{H}_{29} \mathrm{~N}_{2} \mathrm{O}_{5}(\mathrm{M}+\mathrm{H})^{+}, \mathrm{m} / \mathrm{z}$ 497.2076, found 497.2079.

Diethyl 1-benzyl 5-fluoro $\left(3 R, 4^{\prime} R, 5^{\prime} R\right)-2$-oxo-5'-phenyl-4',5'-dihydrospiro[indoline-3,3'pyrrole]-4',5' - dicarboxylate (5b). ${ }^{1} \mathrm{H}-\mathrm{NMR}\left(400 \mathrm{MHz}, \mathrm{CDCl}_{3}\right) \delta 7.68(\mathrm{~d}, J 7.6 \mathrm{~Hz}, 2 \mathrm{H}), 7.51$ $(\mathrm{s}, 1 \mathrm{H}), 7.42-7.30(\mathrm{~m}, 8 \mathrm{H}), 6.93(\mathrm{t}, J 8.4 \mathrm{~Hz}, 2 \mathrm{H}), 6.67$ (q, J 4.0 Hz, 1H), $4.94(\mathrm{~d}, J 3.6 \mathrm{~Hz}, 2 \mathrm{H})$, 4.82 (s, 1H), 4,32 (q, J 7.2 Hz, 2H), 3.67-3.61 (m, 2H), 1.28 (t, J 7.2 Hz, 3H), 0.81 (t, J 7.2 Hz,

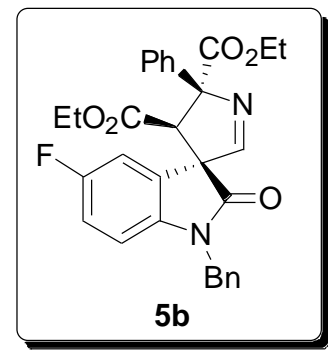

Chemical Formula: $\mathrm{C}_{30} \mathrm{H}_{27} \mathrm{FN}_{2} \mathrm{O}_{5}$ Exact Mass: 514.1904

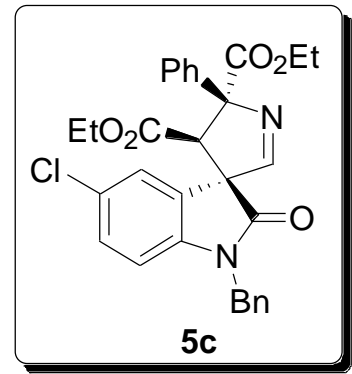

Chemical Formula: $\mathrm{C}_{30} \mathrm{H}_{27} \mathrm{CIN}_{2} \mathrm{O}_{5}$ Exact Mass: 530.1608
$3 \mathrm{H}) .{ }^{13} \mathrm{C}-\mathrm{NMR}\left(100 \mathrm{MHz}, \mathrm{CDCl}_{3}\right) \delta 173.05,171.31,167.73,162.53$, $160.24,139.57,138.72,134.82,129.00,128.23,128.20,128.03,127.25$, $126.53,125.27,125.18,116.33,116.10,115.27,115.01,110.08,110.01$, 89.51, 68.54, 62.52, 59.34, 44.50, 13.97, 13.48. HPLC: Chiralpak OD-H (hexane $/ \mathrm{i}-\mathrm{PrOH}=95 / 5$, flow rate $1 \mathrm{~mL} / \mathrm{min}, \lambda=210 \mathrm{~nm}$ ), $\mathrm{t}_{\mathrm{R}}($ minor $)=29.6$ $\min , \mathrm{t}_{\mathrm{R}}($ major $)=40.7 \mathrm{~min} ; 99 \%$ ee. $[\alpha]_{\mathrm{D}}^{21}=-81.9\left(\mathrm{c}=1.1, \mathrm{CH}_{2} \mathrm{Cl}_{2}\right)$. HRMS (ESI) calcd for $\mathrm{C}_{30} \mathrm{H}_{28} \mathrm{FN}_{2} \mathrm{O}_{5}(\mathrm{M}+\mathrm{H})^{+}$, m/z 515.1982, found 515.1979.

\section{Diethyl 1-benzyl 5-chloro $\quad\left(3 R, 4^{\prime} R, 5^{\prime} R\right)$-2-oxo-5'-phenyl-4',5'-} dihydrospiro [indoline-3,3'-pyrr ole]-4',5'-dicarboxylate (5c). ${ }^{1} \mathrm{H}-\mathrm{NMR}$ $\left(400 \mathrm{MHz}, \mathrm{CDCl}_{3}\right) \delta 7.69(\mathrm{~d}, J 7.2 \mathrm{~Hz}, 2 \mathrm{H}), 7.51(\mathrm{~s}, 1 \mathrm{H}), 7.42-7.27(\mathrm{~m}$, $10 \mathrm{H}), 7.63(\mathrm{~d}, J 8.4 \mathrm{~Hz}, 1 \mathrm{H}), 4.93(\mathrm{~s}, 2 \mathrm{H}), 4.80(\mathrm{~s}, 1 \mathrm{H}), 4.35-4.27(\mathrm{~m}, 2 \mathrm{H})$, $3.70-3.58(\mathrm{~m}, 2 \mathrm{H}), 1.28(\mathrm{t}, J 7.2 \mathrm{~Hz}, 3 \mathrm{H}), 0.81(\mathrm{t}, J 7.2 \mathrm{~Hz}, 3 \mathrm{H}) .{ }^{13} \mathrm{C}-\mathrm{NMR}$ $\left(100 \mathrm{MHz}, \mathrm{CDCl}_{3}\right) \delta 172.77,171.28,167.71,162.36,142.66,138.81$, $134.63,132.68,130.02,129.04,128.25,128.19,128.08,127.22,126.47$, $125.68,115.67,110.98,89.48,68.17,62.53,60.96,59.65,44.42,13.98$, 13.51. HPLC: Chiralpak OD-H (hexane/i-PrOH $=90 / 10$, flow rate 1 
$\mathrm{mL} / \mathrm{min}, \lambda=210 \mathrm{~nm}), \mathrm{t}_{\mathrm{R}}($ minor $)=19.4 \mathrm{~min}, \mathrm{t}_{\mathrm{R}}($ major $)=25.1 \mathrm{~min} ; 97 \%$ ee. $[\alpha]_{\mathrm{D}}{ }^{21}=-17.5(\mathrm{c}=$ 1.1, $\mathrm{CH}_{2} \mathrm{Cl}_{2}$ ). HRMS (ESI) calcd for $\mathrm{C}_{30} \mathrm{H}_{28} \mathrm{ClN}_{2} \mathrm{O}_{5}(\mathrm{M}+\mathrm{H})^{+}, \mathrm{m} / \mathrm{z}$ 531.1687, found 531.1683.

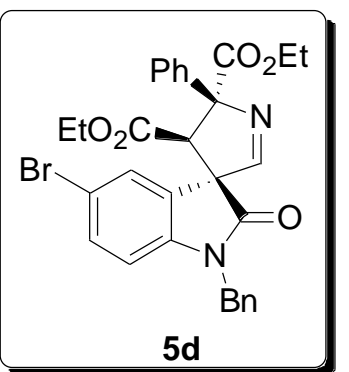

Chemical Formula: $\mathrm{C}_{30} \mathrm{H}_{27} \mathrm{BrN}_{2} \mathrm{O}_{5}$ Exact Mass: 574.1103

Diethyl 1-benzyl 5-bromo $\left(3 R, 4^{\prime} R, 5^{\prime} R\right)-2$-oxo-5'-phenyl-4',5'dihydrospiro[indoline-3,3'-pyrrole]-4',5'-dicarboxylate (5d). ${ }^{1} \mathrm{H}$-NMR $\left(400 \mathrm{MHz}, \mathrm{CDCl}_{3}\right) \delta 7.68(\mathrm{~d}, J 7.2 \mathrm{~Hz}, 2 \mathrm{H}), 7.51(\mathrm{~s}, 1 \mathrm{H}), 7.42-7.27$ (m, $8 \mathrm{H}), 7.21-7.18(\mathrm{~m}, 2 \mathrm{H}), 6.67(\mathrm{~d}, J 8.4 \mathrm{~Hz}, 1 \mathrm{H}), 4.93(\mathrm{~s}, 2 \mathrm{H}), 4.80(\mathrm{~s}, 1 \mathrm{H})$, 4.34-4.29 (m, 2H), 3.68-3.60 (m, 2H), $1.28(\mathrm{t}, J 7.2 \mathrm{~Hz}, 3 \mathrm{H}), 0.81(\mathrm{t}, \mathrm{J} 7.2$ $\mathrm{Hz}, 3 \mathrm{H}) .{ }^{13} \mathrm{C}-\mathrm{NMR}\left(100 \mathrm{MHz}, \mathrm{CDCl}_{3}\right) \delta 172.88,171.29,167.71,162.38$, $142.16,138.77,134.66,129.77,129.03,128.46,128.25,128.19,128.07$, $127.34,127.22,126.48,125.33,110.46,89.50,68.26,62.53,59.56,44.46$, 13.98, 13.48. HPLC: Chiralpak OD-H (hexane/i-PrOH $=90 / 10$, flow rate $1 \mathrm{~mL} / \mathrm{min}, \lambda=210 \mathrm{~nm}), \mathrm{t}_{\mathrm{R}}($ minor $)=19.5 \mathrm{~min}, \mathrm{t}_{\mathrm{R}}($ major $)=26.6 \mathrm{~min}$; $98 \%$ ee. $[\alpha]_{\mathrm{D}}{ }^{21}=-25.5\left(\mathrm{c}=1.0, \mathrm{CH}_{2} \mathrm{Cl}_{2}\right)$. HRMS (ESI) calcd for $\mathrm{C}_{30} \mathrm{H}_{28} \mathrm{BrN}_{2} \mathrm{O}(\mathrm{M}+\mathrm{H})^{+}, \mathrm{m} / \mathrm{z}$ 575.1182, found 575.1180.

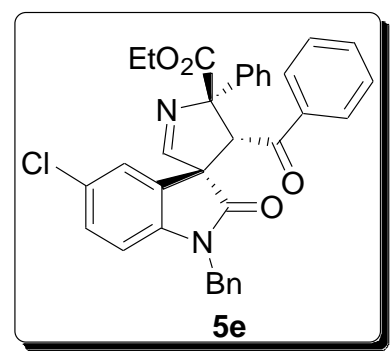

Chemical Formula: $\mathrm{C}_{34} \mathrm{H}_{27} \mathrm{ClN}_{2} \mathrm{O}_{4}$ Exact Mass: 562.1659

Ethyl $4^{\prime}$-benzoyl-1-benzyl 5-chloro $\left(3 R, 4^{\prime} R, 5^{\prime} S\right)-2-0 x 0-5^{\prime}$-phenyl$\mathbf{4}^{\prime}, 5^{\prime}$-dihydrospiro[indoline $\quad \mathbf{- 3 , 3}$-pyrrole $]-5^{\prime}$-carboxylate $(5 \mathrm{e}) .{ }^{1} \mathrm{H}$ NMR (400 MHz, $\left.\mathrm{CDCl}_{3}\right) \delta$ 7.71-7.85 (m, 2H), 7.51-7.47 (m, 4H), 7.36$7.27(\mathrm{~m}, 8 \mathrm{H}), 7.16-7.06(\mathrm{~m}, 4 \mathrm{H}), 6.53(\mathrm{~d}, \mathrm{~J} 8.4 \mathrm{~Hz}, 1 \mathrm{H}), 5.97(\mathrm{~s}, 1 \mathrm{H})$, $5.02(\mathrm{~d}, J 15.6 \mathrm{~Hz}, 1 \mathrm{H}), 4.82(\mathrm{~d}, J 15.6 \mathrm{~Hz}, 1 \mathrm{H}), 4.38-4.31(\mathrm{~m}, 2 \mathrm{H}), 1.28$ $(\mathrm{t}, J$ J $7.2 \mathrm{~Hz}, 3 \mathrm{H}) .{ }^{13} \mathrm{C}-\mathrm{NMR}\left(100 \mathrm{MHz}, \mathrm{CDCl}_{3}\right) \delta 195.52,173.45$, $172.23,162.32,141.55,138.30,138.14,134.64,133.47,129.57,128.98$, $128.69,128.65,128.32,128.24,128.05,128.00,127.01,126.51,124.69$, $110.25,91.07,69.53,62.69,57.79,50.84,44.33$, 13.95. HPLC: Chiralpak AD-H (hexane/i-PrOH $=80 / 20$, flow rate $1 \mathrm{~mL} / \mathrm{min}, \lambda=210 \mathrm{~nm}$ ), $\mathrm{t}_{\mathrm{R}}$ (major) $=22.5$ min, $t_{R}($ minor $)=43.5 \mathrm{~min} ;>99 \%$ ee. $[\alpha]_{\mathrm{D}}{ }^{21}=-44.7\left(\mathrm{c}=1.2, \mathrm{CH}_{2} \mathrm{Cl}_{2}\right)$. HRMS (ESI) calcd for $\mathrm{C}_{34} \mathrm{H}_{28} \mathrm{ClN}_{2} \mathrm{O}_{4}(\mathrm{M}+\mathrm{H})^{+}, m / z$ 563.1738, found 563.1735.

\section{Acknowledgements}

Financial support from the National Natural Science Foundation of China (NSFC-21373073) and the Program for ChangJiang Scholars and Innovative Research Team in Chinese University (IRT 1231 ) is gratefully acknowledged. G.Z. appreciated a QianJiang Scholar from ZheJiang Province in China.

\section{References}

1. Lin, H.; Danishefsky, S. J. Angew. Chem., Int. Ed. 2003, 42, 36. http://dx.doi.org/10.1002/anie.200390048 
2. Galliford, C. V.; Scheidt, K. A. Angew. Chem., Int. Ed. 2007, 46, 8748.

http://dx.doi.org/10.1002/anie.200701342

PMid:17943924

3. Marti, C.; Carreira, E. M. Eur. J. Org. Chem. 2003, 2209.

http://dx.doi.org/10.1002/ejoc.200300050

4. Franz, A. K.; Dreyfuss, P. D.; Schreiber, S. L. J. Am. Chem. Soc. 2007, 129, 1020.

http://dx.doi.org/10.1021/ja067552n

PMid:17263369

5. Ding, K.; Lu, Y; Nikolovska-Koleska, Z.; Qiu, S.; Ding, Y.; Gao, W.; Stuckey, J.; Roller, P.

P.; Tomita, Y.; Deschamps, J. R.; Wang, S. J. Am. Chem. Soc. 2005, 127, 10130.

http://dx.doi.org/10.1021/ja051147z

PMid:16028899

6. Shangary, S.; Qin, D.; McEachern, D.; Liu, M.; Miller, R. S.; Qiu, S.; Nikolovska-Coleska, Z.; Ding, K.; Wang, G.; Chen, J.; Bernard, D.; Zhang, J.; Lu, Y.; Gu, Q.; Shah, R. B.; Pienta, K. J.; Ling, X.; Kang, S.; Guo, M.; Sun, Y.; Yang, D.; Wang, S. Proc. Natl. Acad. Sci. U.S.A. 2008, 105, 3933.

http://dx.doi.org/10.1073/pnas.0708917105

PMid:18316739 PMCid:PMC2268798

7. Nicolaou, K. C.; Snyder, S. A. Proc. Natl. Acad. Sci. USA, 2004, 101, 11929.

http://dx.doi.org/10.1073/pnas.0403799101

PMid:15302925 PMCid:PMC514411

8. Corey, E. J. Angew. Chem., Int. Ed. 2002, 41, 1650.

http://dx.doi.org/10.1002/1521-3773(20020517)41:10<1650::AID-ANIE1650>3.0.CO;2-B

9. Trost, B. M.; Cramer, N.; Silverman, S. M. J. Am. Chem. Soc. 2007, 129, 12396.

http://dx.doi.org/10.1021/ja075335w

PMid:17880222 PMCid:PMC2615581

10. Chen, X.; Wei, Q.; Luo, S.; Xiao, H.; Gong, L. J. Am. Chem. Soc. 2009, 131, 13819.

http://dx.doi.org/10.1021/ja905302f

PMid:19736987

11. Voituriez, A.; Pinto, N.; Neel, M.; Retailleau, P.; Marinetti, A. Chem.-Eur. J. 2010, 16, 12541.

http://dx.doi.org/10.1002/chem.201001791

PMid:20853298

12. Liu, Y.; Nappi, M.; Arceo, E.; Vera, S.; Melchiorre, P. J. Am. Chem. Soc. 2011, 133, 15212. http://dx.doi.org/10.1021/ja206517s

PMid:21842900

13. Tan, B.; Hernandez-Torres, G.; Barbas, C. F., III, J. Am. Chem. Soc. 2011, 133, 12354.

http://dx.doi.org/10.1021/ja203812h

PMid:21780763

14. Peng, J.; Huang, X.; Jiang, L.; Cui, H.; Chen, Y. Org. Lett. 2011, 13, 4584. 
http://dx.doi.org/10.1021/ol201776h

PMid:21815615

15. Tan, B.; Candeias, N. R.; Barbas, C. F., III. Nat. Chem. 2011, 3, 473.

PMid:21602863

16. Tan, B.; Candeias, N. R.; Barbas, C. F., III. J. Am. Chem. Soc. 2011, 133, 4672.

http://dx.doi.org/10.1021/ja110147w

PMid:21395245

17. Tan, B. Hernandez-Torres, G.; Barbas, C. F., III. J. Am. Chem. Soc. 2011, 133, 12354.

http://dx.doi.org/10.1021/ja203812h

PMid:21780763

18. Zhong, F.; Han, X.; Wang, Y.; Lu, Y. Angew. Chem., Int. Ed. 2011, 50, 7837.

http://dx.doi.org/10.1002/anie.201102094

PMid:21728218

19. Jia, Z.; Jiang, H.; Li, J.; Gschwend, B.; Li, Q.; Yin, X.; Grouleff, J.; Chen, Y.; Jorgensen, K. A. J. Am. Chem. Soc. 2011, 133, 5053.

http://dx.doi.org/10.1021/ja1112194

PMid:21405125

20. Cao, Y.; Jiang, X.; Liu, L.; Shen, F.; Wang, R. Angew. Chem., Int. Ed. 2011, 50, 9124.

http://dx.doi.org/10.1002/anie.201104216

PMid:21919145

21. Tan, B.; Zeng, X.; Leong, W. W. Y.; Shi, Z.; Barbas, C. F., III.; Zhong, G., Chem.-Eur. J. $2012,18,63$.

http://dx.doi.org/10.1002/chem.201103449

PMid:22162076

22. Bui, T.; Candeias, N. R.; Barbas, C. F., III, J. Am. Chem. Soc. 2010, 132, 5574.

http://dx.doi.org/10.1021/ja101032j

PMid:20356308

23. He, R.; Shirakawa, S.; Maruoka, K. J. Am. Chem. Soc. 2009, 131, 16620.

http://dx.doi.org/10.1021/ja906821y

PMid:19886657

24. Quaternary Stereocenters. Challenges and Solutions in Organic Synthesis (Eds.: Christoffers, Baro, J. A.), Wiley-VCH, Weinheim, 2006.

25. Douglas,C. J.; Overman, L. E. Proc. Natl. Acad. Sci. USA, 2004, 101, 5363.

http://dx.doi.org/10.1073/pnas.0307113101

PMid:14724294 PMCid:PMC397386

26. Tian, S.; Chen, Y.; Hang, J.; Tang, L.; McDaid, P.; Deng, L. Acc. Chem. Res. 2004, 37, 621. http://dx.doi.org/10.1021/ar030048s

PMid:15311961

27. Li, H.; Wang, Y.; Tang, L.; Deng, L., J. Am. Chem. Soc. 2004, 126, 9906. 
http://dx.doi.org/10.1021/ja0472811

PMid:15303849

28. Ye, J.; Dixon, D. J.; Hynes, P. S. Chem. Commun. 2005, 4481.

http://dx.doi.org/10.1039/b508833j

PMid:16136258

29. McCooey, S. H.; Connon. S. J. Angew. Chem., Int. Ed. 2005, 44, 6367.

http://dx.doi.org/10.1002/anie.200501721

PMid:16136619

30. Vakulya, B.; Varga, S.; Csampai, A.; Soos, T., Org. Lett. 2005, 7, 1967.

http://dx.doi.org/10.1021/ol050431s

PMid:15876031

31. Mattson, A. E.; Zuhl, A. M.; Reynolds, T. E.; Scheidt, K. A. J. Am. Chem. Soc. 2006, 128, 4932.

http://dx.doi.org/10.1021/ja056565i

PMid:16608309

32. Tan, B.; Chua, P. J.; Li, Y.; Zhong, G. Org. Lett. 2008, 10, 2437.

http://dx.doi.org/10.1021/ol8007183

PMid:18489178

33. Tan, B.; Shi, Z.; Chua, P. J.; Zhong,G. Org. Lett. 2008, 10, 3425.

http://dx.doi.org/10.1021/ol801246m

PMid:18616339

34. Tan, B.; Chua, P. J.; Zeng, X.; Lu, M.; Zhong, G. Org. Lett. 2008, 10, 3489.

http://dx.doi.org/10.1021/ol801273x

PMid:18630924

35. Tan, B.; Zhang, X.; Chua, P. J.; Zhong, G. Chem. Commun. 2009, 779.

http://dx.doi.org/10.1039/b813915f

PMid:19322439

36. Tan,B.; Lu,Y.; Zeng, X.; Chua, P. J.; Zhong, G. Org. Lett. 2010, 12, 2682.

http://dx.doi.org/10.1021/ol1007795

PMid:20469881

37. Wu,Y.; Singh, R. P.; Deng, L. J. Am. Chem. Soc., 2011, 133, 12458.

http://dx.doi.org/10.1021/ja205674x

PMid:21766859 PMCid:PMC3156085

38. Dalko, P. I.; Moisan, L. Angew. Chem., Int. Ed. 2004, 43, 5138.

http://dx.doi.org/10.1002/anie.200400650

PMid:15455437

39. List, B. Chem. Commun. 2006, 819.

http://dx.doi.org/10.1039/b514296m

PMid:16479280 
40. Dondoni, A.; Massi, A. Angew. Chem., Int. Ed. 2008, 47, 4638.

http://dx.doi.org/10.1002/anie.200704684

PMid:18421733

41. Melchiorre, P.; Marigo, M.; Carlone, A.; Bartoli, G. Angew. Chem., Int. Ed. 2008, 47, 6138. http://dx.doi.org/10.1002/anie.200705523

PMid:18666089

42. Barbas, C. F., III. Angew. Chem., Int. Ed. 2008, 47, 42.

http://dx.doi.org/10.1002/anie.200702210

PMid:17943929

43. Ito, Y.; Samura, M.; Hayashi, T. J. Am. Chem. Soc. 1986, 108, 6405. http://dx.doi.org/10.1021/ja00280a056

44. Song, J.; Guo, C.; Chen, P.; Yu, J.; Luo, S. ; Gong, L. Chem.-Eur. J. 2011, 17, 7786.

http://dx.doi.org/10.1002/chem.201100636

PMid:21618634

45. SladoJevich, F.; Trabocchi, A.; Guarna, A.; Dixon, D. J. J. Am. Chem. Soc. 2011, 133, 1710. http://dx.doi.org/10.1021/ja110534g

PMid:21247165

46. Wang, L.; Bai, J.; Peng, L.; Qi, L.; Jia, L.; Guo, Y.; Luo, X.; Xu, X.; Wang, L. Chem. Commun. 2012,48, 5175.

http://dx.doi.org/10.1039/c2cc30746d

PMid:22517246

47. Tan, D. Nat. Chem. Biol. 2005, 1, 74.

http://dx.doi.org/10.1038/nchembio0705-74

PMid:16408003

48. Kissane, M.; Maguire, A. Chem. Soc. Rev. 2010, 39, 845.

http://dx.doi.org/10.1039/b909358n

PMid:20111795

49. We only determined the relative configuration of the Bn-protected products based on 2D NMR (NOESY). 